\title{
Computed Tomography and Position Emission Tomography in Radiation Oncology
}

\section{Sasa Mutic, MS}

\author{
Associate Professor, Radiation Oncology, Siteman Cancer Center, Mallinckrodt Institute of Radiology, \\ Washington University School of Medicine
}

DOI: $10.17925 /$ OHR.2005.00.00.1b

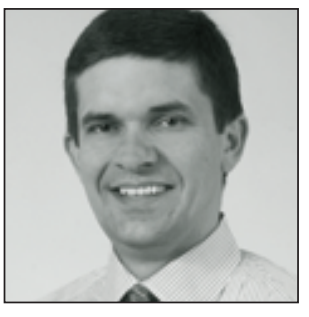

Sasa Mutic, MS, a radiation therapy medical physicist, is Associate Professor of Radiation Oncology at the Mallinckrodt Institute of Radiology at the Washington University School of Medicine in St Louis, Missouri. His main clinical and research interests include implementation of different medical imaging modalities in the

radiotherapy treatment planning and delivery process. He has written and lectured extensively on this topic. He was the Co-chair of the Task Group on CT-Simulation Quality Assurance of the Radiation Therapy Committee of the American Association of Physicists in Medicine, and actively participates in editorial activities for several medical journals. Mr Mutic received

his graduate degree in medical physics from the University of

Colorado, and completed a medical physics residency at the Washington University School of Medicine. He is certified in therapeutic radiological physics by the American Board of Radiology.

\section{Introduction}

During the 1990s, the radiation oncology community made many improvements in its ability to accurately deliver high doses of radiation to complex tumor shapes. Modern treatment planning systems can calculate relatively efficiently optimized treatment plans to complex target volumes, and treatment machines can deliver these plans with high precision in short treatment times. Figure 1 shows a radiation dose distribution for the treatment of a para-nasal tumor with brain invasion. Radiation doses (shown in color wash) conform closely to the tumor volume while sparing the surrounding critical organs. Similar dose distributions can be achieved for some other cancer sites as well. The ability to deliver radiation doses with small uncertainties (in both location and magnitude) increases the need to accurately define anatomical location and the extent of tumor volumes, and to determine biological properties of individual tumors. A better understanding of tumor extent and biology can result in improved radiation dose distributions delivered to patients, which should translate to better outcomes and/or reduced complications. Medical imaging is one of the main tools used in radiation therapy (RT) for disease detection, staging, treatment modality selection, tumor volume definition, radiotherapy treatment planning, and prognosis and follow-up. Therefore, the data contained in patient images profoundly affects patient management and delivery of radiation.

Research and development (R\&D) in medical imaging offers one of the greatest avenues for improvement in radiation oncology treatments. The ultimate goal for medical imaging in RT is to allow radiation oncologists to accurately delineate and biologically characterize an individual tumor, select an appropriate course of therapy, and predict tumor response as early as possible. To biologically characterize an individual tumor means that an imaging modality does not need to image gross anatomically visible changes; rather, it must capture information about a tumor's underlying physiology, metabolism, function, and molecular makeup. Therefore, imaging information used in RT can be classified as anatomical or biological, and the radiation oncologist relies on multiple imaging modalities (multi- modality imaging) to gather this information. Four primary imaging modalities are used in $\mathrm{RT}$ :

- computed tomography (CT);

- magnetic resonance (MR);

- ultrasound (US); and

- nuclear medicine (primarily positron emission tomography (PET)).

CT and US provide primarily anatomical information, while PET and MR can provide biological as well as anatomical information. This article describes the current state of CT and PET use in RT. The main purpose of imaging in radiation oncology today is to gather anatomical information. The vast majority of studies are performed with CT, and CT will remain the primary imaging modality in radiation oncology for the foreseeable future. However, imaging of functional and biological tumor properties is increasing and could change many RT practices by improving disease detection, staging, therapy selection, target design, prognosis, and follow-up. Thus far, PET has been the primary imaging modality used for this purpose.

The Potential of PET Imaging in Radiation Therapy

PET has been used for the management of RT patients on a limited scale for many years. This interest has grown significantly in the past five years and the number of publications on this topic has also seen a rapid increase. While it is still unclear as to what can be achieved with PET in RT, the following are some of the processes that have been shown to be affected.

\section{Detection}

By detecting changes in the molecular and biochemical process, biological imaging can demonstrate disease before it becomes anatomically detectable with CT or MR imaging (MRI). Changes in tumor detection capabilities can lead to modification in RT volumes and dose prescriptions. 
Staging

PET has affected patient staging in several treatment sites (lung, cervix, colon, etc.). ${ }^{1}$ Better knowledge of the true extent of the patient's disease can significantly alter patient management. For some patients, who would otherwise undergo curative radiotherapy, PET may demonstrate distal disease or alter the extent of local disease and indicate that a palliative course of therapy is more appropriate. These patients would not only be spared the side effects of a futile curative treatment, but the overall healthcare costs could also be lowered due to PET findings.

In addition to better staging, it has also been suggested that PET may improve information about individual tumor biology (phenotype). This would allow further stratification of patients within the same clinical stage. So rather than basing therapy selection for an individual patient on the stage alone, which is statistically appropriate for a large group of patients, biological properties of an individual tumor can then be used for therapy selection. The tumor phenotype information may affect inter- and intra-modality patient management depending on suspected radiation or chemotherapy sensitivity of an individual tumor.

Target Definition and Altered

Dose Distributions

As stated above, the true extent of the disease may extend beyond anatomically defined volumes on CT or MR, and PET has already been shown to be a valuable tool for defining the extent of target volumes. Ling et $\mathrm{al}^{2}$ described the concept of biological target volumes (BTVs) where portions of tumor volumes are assigned increased importance based on imaging data. The identification of BTVs would be based on unique properties of imaged tumor areas with respect to proposed therapy, such as an increased radioresistance of tumor cells. It is possible that the concept of BTVs can be implemented with PET and that radiation doses can be escalated to these BTVs if deemed necessary. For example, Chao et al. $^{3}$ have shown how PET imaging with $\mathrm{Cu}(\mathrm{II})$-diacetyl-bis $\left(\mathrm{N}_{4}\right.$-methylthiosemicarbazone) (Cu-ATSM) tracer can be used to identify hypoxic volumes for head and neck tumors. Hypoxic areas, which are associated with increased radioresistance, would, in this case, become BTVs. Advanced RT
Figure I: Color-wash Representation of Radiation

Dose Distribution for Paranasal Tumor with

Brain Invasion

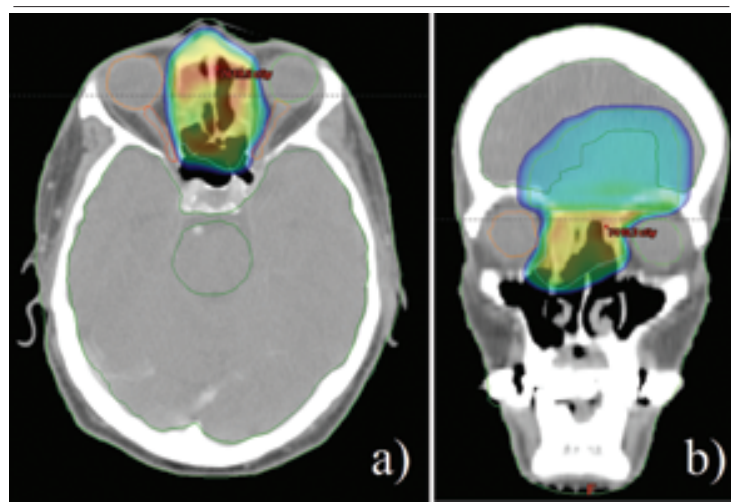

a) axial view, b) frontal (coronal) view. Blue colors represent lower radiation doses and red colors represent higher doses.

\section{Figure 2}
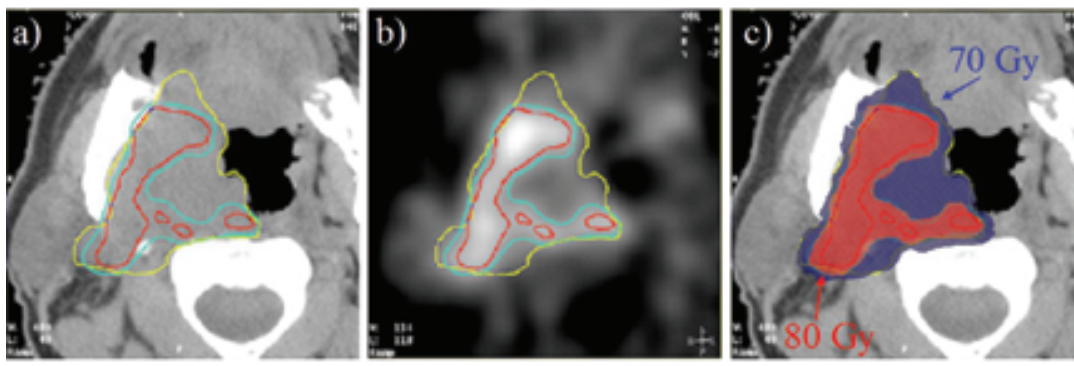

a) CT image with a delineation of tumor target volume (yellow line) and overlaid area of 60Cu-ATSM intensity from PET image (red line) representing presumably area of tumor hypoxia (BTV), b) PET image with a delineation of Cu-ATSM intensity and tumor hypoxia (red line) and the overlaid target volume as defined on CT (yellow line), c) a hypothetical RT plan which delivers 80 Gy to the radioresistant hypoxic portion of the tumor or the BTV (red area) and 70 Gy to the rest of the target volume.

treatment planning and delivery techniques can then be used to deliver escalated radiation doses to these volumes. Figure 2 shows a CT, Cu-ATSM-based PET and RT treatment plan images for a patient with a hypoxic BTV inside the tumor.

Evaluation of Response to Therapy and

Follow-up

Currently, tumor control and effectiveness of radiotherapy is evaluated in the weeks and months following the completion of treatments. The evaluation, similar to detection and diagnosis, relies largely on anatomical changes, which take time to manifest. With this approach, by the time it is determined that the therapy was not effective and that local tumor control has not been achieved it may be too late to initiate a second line of

1. Dizendorf E V, Baumert B G, von Schulthess G K, Lutolf $U$ M and Steinert H C, "Impact of whole-body 18F-FDG PET on staging and managing patients for radiation therapy”, J. Nuc. Med. (2003), 44: pp. 24--29.

2. Ling C C, Humm J, Larson S, Amols H, Fuks Z, Leibel S and Koutcher J A, "Towards multidimensional radiotherapy (MDCRT): Biological imaging and biological conformality”, Int. J. Radiat. Oncol. Biol. Phys. (2000), 47: pp. 551-560.

3. Chao K S C, Bosch W R, Mutic S, Lewis J S, Dehdashti F, Mintun M A, Dempsey J F, Perez C A, Purdy J A and Welch M $J$, "A novel approach to overcome hypoxic tumor resistance Cu-ATSM-Guided intensity-modulated radiation therapy", Int. J. Radiat. Oncol. Biol. Phys. (2001), 49: pp. 1,171-1,182. 
therapy. A developing area of PET imaging is the monitoring of tumor response to therapy. PET may be able to detect changes (or no change) in the tumor sooner in the course of RT patient management than anatomical imaging and allow modifications in the proposed course of therapy if necessary. This application of PET imaging, while promising, has several potential limitations due to over-estimation or under-estimation of tumor uptake. Jerusalem et al. ${ }^{4}$ noted that the presence of inflammatory cells can result in elevated imaging agent uptake even with successful response to therapy. In certain situations these cells may have an even higher uptake than viable tumor cells. Conversely, poor PET resolution may prevent detection of small residual tumor volumes.

Technology Overview

One of the major changes in RT imaging is the approach of imaging equipment manufacturers to radiation oncology and its unique imaging needs. Radiotherapy imaging has special needs with respect to scanner geometry, patient positioning, and target delineation. These needs are often not a significant concern in diagnostic radiology. The scanners used in RT should have flat table tops, larger openings to accommodate patients in treatment positions that will facilitate minimal irradiation of normal anatomy, and software tools that can improve patient positioning and target delineation. Until the late 1990s, imaging equipment was designed and manufactured, almost exclusively, with diagnostic radiology needs in mind, and these devices would then have to be modified for therapy scanning. Frequently, there were significant limitations. In the past few years there have been a number of scanners introduced on the market with features designed specifically for radiotherapy. Radiotherapy needs have begun to drive product development. This change in manufacturer approach to radiotherapy has resulted in a variety of imaging options available to radiation oncology departments and it has improved their ability to image patients for treatment planning purposes. The two most significant changes in CT technology for RT scanning purposes in recent years have been the introduction of a larger gantry bore opening (large bore $\mathrm{CT})^{5}$ and multislice image acquisition (multi-slice CT). ${ }^{6}$

Large Bore CT

Large bore CT scanners were specifically designed with radiation therapy needs in mind. One of the requirements in the treatment of several cancer sites (breast, lung, vulva, etc.) is for extremities to be positioned away from the torso during treatment and therefore during the treatment planning scan. When acquiring a CT scan with a patient in such a position, extremities often cannot fit through a conventional $70 \mathrm{~cm}$ diameter CT scanner bore opening. In these situations, patient positioning needs to be modified in order to acquire the scan. This can result in a less than optimal treatment position (the patient may be less comfortable and therefore the daily set-up reproducibility may be compromised). Large bore scanners $(80-85 \mathrm{~cm}$ opening) have virtually eliminated these problems. These scanners also have an increased scan field of view (SFOV) compared with conventional units. The SFOV determines the maximum size of the patient that can completely fit in the CT image. For RT treatment planning purposes it is necessary to have the full extent of the patient's skin on the CT image, this is required for accurate calculation of radiation doses. Conventional CT scanners typically have $48 \mathrm{~cm}$ SFOV, meaning that the skin cannot be imaged for patients who are larger in size than $48 \mathrm{~cm}$. This is a significant problem for treatment of obese patients. Depending on the manufacturer, large bore scanners offer SFOV between 60 and $82 \mathrm{~cm}$, which is a significant improvement.

Multi-slice CT

The CT scanner that could acquire two images (slices) simultaneously was first introduced in 1992. Since then, multi-slice CT has gained wide-spread acceptance and scanners which can acquire four, eight, 10, 16, 32, 40, 64, etc. images simultaneously are now available. Compared with single-slice scanners, multi-slice scanners acquire images several times faster and they can also scan longer volumes with thinner image thickness. One shortcoming of planning RT for tumors located in the thorax and in the upper abdomen using a single-slice CT scanner is that the tumor motion cannot be appreciated on these images. Therefore, it is difficult to target radiation to the tumor volumes without irradiating relatively large volumes of the surrounding healthy tissues. A recent topic of research has been four-dimensional (4-D) or five-dimensional (5D) CT imaging using multi-slice CT technology. ${ }^{7}$ Using specialized acquisition techniques and multi-slice scanners, a series of images can be acquired that can demonstrate tumor motion as a function of patient breathing. This information can then be used to conform radiation doses closer to target volumes and increase the sparing of surrounding healthy tissues while possibly increasing doses to tumor volumes.

4. Jerusalem G, Hustinx R, Beguin Y and Fillet G, "PET scan imaging in oncology", Eur. J. Cancer (2003), 39:pp. 1,525-1,534.

5. Garcia-Ramirez J L, Mutic S, Dempsey J F, Low D A and Purdy J A, "Performance evaluation of an 85-cm bore x-ray computed tomography scanner designed for radiation oncology and comparison with current diagnostic CT scanners", Int. J. Radiat. Oncol. Biol. Phys. (2002), 52, pp. 1,123-1,131.

6. Klingenbeck-Regn K, Schaller S, Flohr T, Ohnesorge B, Kopp A F and Baum U, "Subsecond multi-slice computed tomography: basics and applications”, Eur. J. Radiol. (1999), 31: pp. 110-124. 
Due to the importance of increased scanner opening size and multi-slice acquisition capabilities for RT treatment planning, several manufacturers have recently introduced large bore multi-slice scanners. These scanners allow efficient scanning of patients in preferred RT treatment positions and should be able to facilitate imaging of tumor motion due to breathing.

\section{PETICT Scanners}

PET images for RT planning can come from a standalone PET scanner or a combined PET/CT unit. A $\mathrm{PET} / \mathrm{CT}$ unit is an imager that combines PET and a CT scanner in a common housing. ${ }^{8}$ The two scanners are still relatively independent but they do share the scanner table, control console, and some other hardware. Combined PET/CT scanners offer several advantages for radiotherapy imaging and are generally preferred over stand-alone units. Registering and correlating tumor information between PET and CT images is much easier if the images are acquired on the same scanner (see Figure 3). The patient position and image location correlate rather closely and PET information can be efficiently associated with the CT anatomy. In the same imaging session a treatment planning CT can be acquired along with PET images, which can be used to evaluate tumor properties.

This technology is greatly increasing the use of PET imaging for RT planning and several such scanners have been installed in radiation oncology departments. One of the reasons for relatively few reports in the literature on the use of PET for RT planning is due to difficult incorporation of PET images from stand-alone scanners. With increased use due to PET/CT scanners, a large number of studies will be able to evaluate whether the use of PET images for target delineation will improve outcomes and/or reduce treatmentrelated complications.

Discussion

One opportunity for an overall improvement of radiation oncology is a better understanding of tumors through biological imaging. Biological imaging has been shown to better demonstrate the extent of disease than anatomical imaging and also to better characterize individual tumor properties. However, numerous studies have also shown that PET imaging (especially with 18F-fluoro-2-deoxyD-glucose (FDG)) can be a source of false positive or false negative findings. Due to this concern, PET imaging is
Figure 3: Images Acquired on a Combined PETICT Scanner
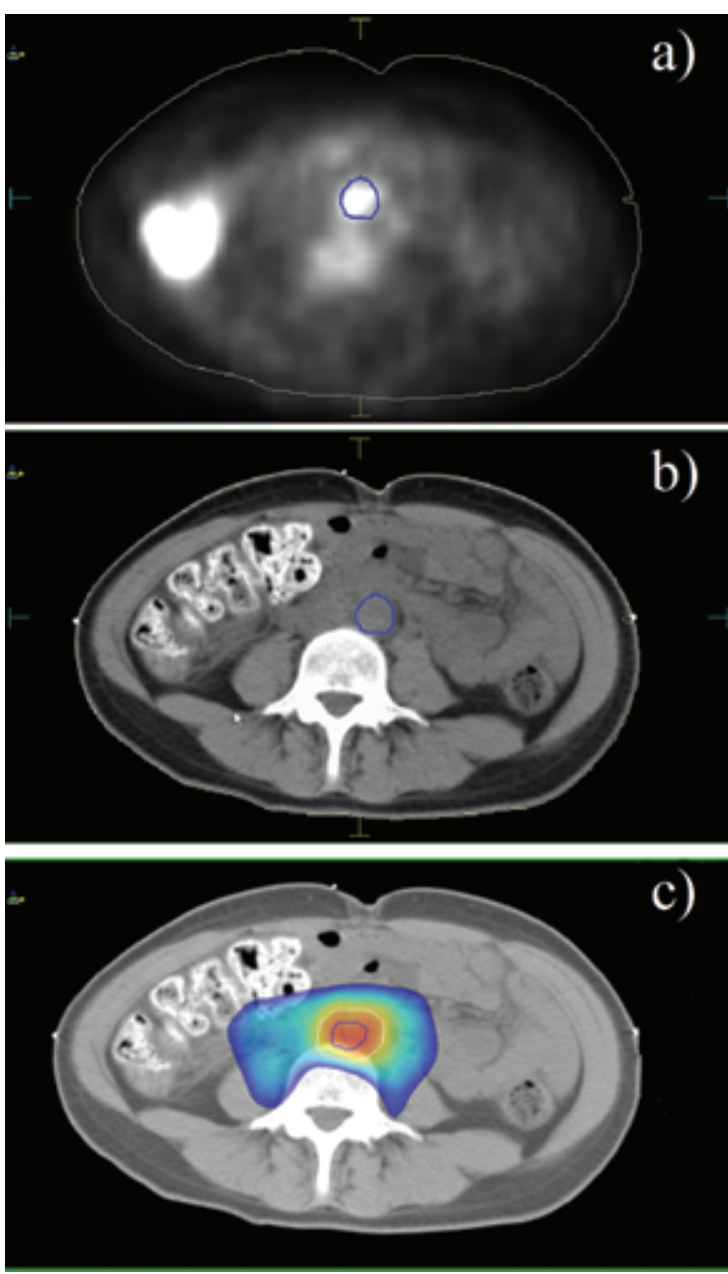

a) PET image from a PET/CT scanner showing a positive para-aortic lymph node for a patient with cervical cancer, b) lymph-node location correlated to the CT scan, c) radiation dose distribution for treatment of the para-aortic lymph node bed. Blue colors represent lower radiation doses and red colors represent higher doses.

probably best used as a complementary tool in the management of radiotherapy patients, especially given the fact that this is a relatively new imaging modality for many radiation oncology practices and that there is a relatively small amount of published data on the use of PET imaging for this purpose. The correlation of FDG uptake with anatomic abnormalities detected by CT or MRI (or some other diagnostic method) provides valuable insight about functional and anatomical properties of a tumor. PET/CT scanners and the availability of registered images simplifies, to an extent, the validation of PET findings and will allow greater use of PET images in radiation oncology.

7. Low D A, Nystrom M, Kalinin E, Parikh P, Dempsey J F, Bradley J D, Mutic S, Wahab S H, Islam T, Christensen G, Politte $D G$ and Whiting $B R$, "A method for the reconstruction of 4-dimensional synchronized CT scans acquired during free breathing", Med. Phys. (2003), 30, pp. 1,254-1,263.

8. Beyer T, Townsend D W, Brun T, Kinahan P E, Charron $M$ and Roddy R, et al, "A combined PET/CT scanner for clinical oncology”, J. Nuc. Med. (2000), 41: pp. 1,369-1,379. 